\title{
Formal Specification of Ontology Networks
}

\author{
Edelweis Rohrer \\ Instituto de Computación, Facultad de Ingeniería, \\ Universidad de la República, Uruguay \\ erohrer@fing.edu . uy
}

\begin{abstract}
Nowadays, it is very often to integrate existing ontologies, combining them in a ontology network to accomplish the requirements of more complex applications. This $\mathrm{PhD}$ research 11 aims to identify and formally define the relationships among the networked ontologies, addressing its use in real applications and taking care of their consistency.
\end{abstract}

Keywords: ontology network, ontology relationships formalization, logical consistency.

\section{Motivation and Research Questions}

Nowadays, autonomously developed ontologies in different domains (health, learning) are used together in complex applications. However, how they are combined is usually hidden in the application code. This situation leads to think on ontology networks as a new engineering concept, which explicitly expresses how ontologies are combined. Let suppose a scenario involving several domains, such as a web resource recommender system (Figure 1). The Resources domain describes web contents queried by users, the Quality domain, the quality assessment process of web resources, the User Context domain, the user profile and context and the Criteria Selection domain, the criteria used to recommend a given resource to a user. In this example, the relationship between Quality and

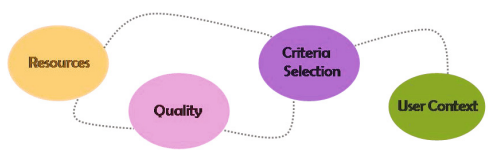

Fig. 1. A Recommendation Ontology Network

Resources domains appears since in this case study, web resources are assessed according to some quality criteria. Then, it is important to explicitly specify not only the semantics of each domain, but moreover adding knowledge about how

\footnotetext{
${ }^{1}$ Tutored by Regina Motz of Instituto de Computación, Facultad de Ingeniería, Universidad de la República, Uruguay and Alicia Díaz of LIFIA, Facultad de Informática, Universidad Nacional de La Plata, Argentina.
} 
these domains are related. The main motivation of this thesis is the identification and formal definition of the different relationships among ontologies, to describe a particular application, keeping the logical consistency. That is, there should not be an axiom of an ontology that causes contradictory results over another ontology in an ontology network. In a real application, the ontology network consistency could be computationally hard to be checked, then, the trade-off between keeping the consistency and taking care of the computational properties, is one of the main issues of this work. The main contribution will be to facilitate developers in the design of ontology networks, expliciting how ontologies can be linked, keeping them as independent components. In the remainder of this paper: Section 2 gives a background overview, Section 3 explains the PhD approach, Section 4 introduces methodology issues and Section 5 presents the work already done.

\section{State of the Art}

According to the presented motivation, I take as starting point the work of Allocca et al. [1], who identify and define general relations between ontologies, such as includedIn and equivalentTo, describing them in the DOOR ontology.

Grau et al. 2] define an $\varepsilon-$ connection as a "set of connected ontologies", introducing link properties, which connect two ontologies. The semantic of these properties is like the useSymbolsOf relationship, defined in the $\mathrm{PhD}$ work. I also based my study in a more recent work of Grau et al. 3, which adapts the notions of module and black-box behavior, to the reuse of ontologies. Konev et al. [4] analize the same concepts and others such as robustness of a query language, based on the concept of inseparability of ontologies. These two works 34 also analise the computational complexity issue for Description Logics (DL) with different expressivity, so, I am taking advantage of their results.

The work of Borgida et. al [5] defines directional links between ontologies, called bridge rules and the concept of distributed T-box, DL T-boxes connected through bridge rules. The bridge rules capture the idea of linking ontologies through subsumption as well as more general relationships, while my work intends to clearly distinguish different ways of connecting ontologies, to make them explicit.

Giunchiglia et al. 6] define the concept of abstraction without relating it to ontologies. However, I take this idea to define the is TheSchemaFor relationship.

There also exist works that define the ontology mapping between concepts, roles and instances [78, taken to formalize the relationship mapsSymbols To.

\section{The Proposed Approach}

I have formalized a set of ontology relationships, which allowed me to design ontology networks for some case studies. For these case studies, this set of relationships was adecuate to explicitly express the links among the different domain ontologies. Next, I give an intuitive description of each ontology relationship.

is AConsistentExtension Of: describes an extension of an ontology by a number of additional axioms. 
usesSymbols $O f$ : this relationship holds when an ontology $O$ needs to be linked to individuals from another ontology $O^{\prime}$, through a property which relates them. mapsSymbols To: an ontology $O$ mapsSymbols To an ontology $O^{\prime}$ if there exists an alignment from $O$ to $O^{\prime}$, covering part of the vocabulary of $O$.

is TheSchemaFor: keeps the link between a model and its meta-model.

In the web resource recommender system introduced in Section 1, the $R e$ sources domain is composed by three ontologies, illustrated in Figure 2 in a simplified version. The main concepts of the WebSite Specification ontology are
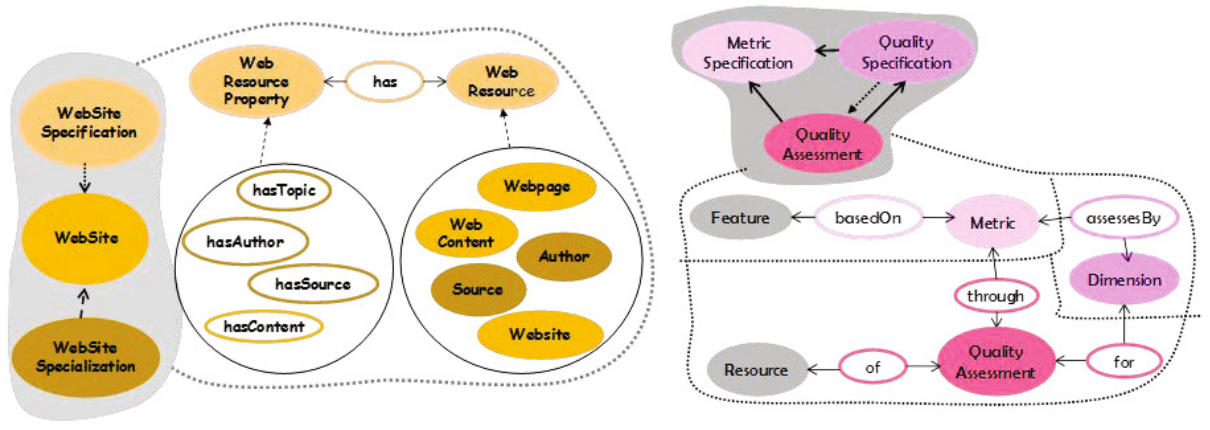

Fig. 2. Resources and Quality domains

WebResource and WebResourceProperty. A web resource is any resource identified by an URL, for instance a webpage. A web resource property models the properties of a web resource, for instance, hasContent and hasAuthor. The WebSite ontology has as main concepts: WebContent, WebPage and WebSite. The WebSite Specialization ontology adds properties to these concepts, such as hasAuthor and hasSource. In the Resources domain the isTheSchemaFor relationship links some ontologies. The WebSite Specification ontology is the metamodel for the Website and WebSite Specialization ontologies, since the concepts and relations of these ontologies are instances of WebResource and WebResourceProperty concepts.

The Quality domain is composed by three ontologies: Metric Specification, Quality Specification and Quality Assessment. They conceptualize metrics, quality assurance specifications and quality assessments. Figure 2 shows a simplified version. Some of these ontologies are related to ontologies of the Resources domain. The mapSymbols To relationship links the Quality Assessment and WebSite ontologies, through an alignment of the Resource and WebContent concepts. This relationship is also used between Metric Specification and WebSite Specification ontologies, mapping the Feature and WebResourceProperty concepts, to specify that a metric is based on some web resource property. Here, it is clear the convenience of having some ontologies that play the role of metamodel for others.

In the formalization of the ontology relationships I consider the language $\mathcal{Q} \mathcal{L}$, which is the selected DL with the adequate expressivity for the application 
to be described. That is, besides the knowledge represented by each ontology, I consider the expressivity required by the application, for the knowledge inference of the ontology network. For a case study, maybe it is enough a DL, for example $\mathcal{A L C}$, and not a more expressive DL like $\mathcal{A L C} \mathcal{Q}$, computationally more expensive 910. I am addressing the study of the logical consistency of the ontology network based on the concept of inseparability introduced by Konev et.al [4], w.r.t. this language $\mathcal{Q L}$ and I am starting to study the computational complexity of the algorithms for checking the consistency. The results obtained will be analized varying the DL expressivity of the $\mathcal{Q} \mathcal{L}$, for the different relationships.

Although my work is inspired on [1], it is different since the main focus in [1] is the detection and definition of ontology relationships in a large ontology repository, while my focus is the identification and DL formalization of a set of ontology relationships, enough to design an ontology network for a particular application. This is done considering the logical consistency of the ontology network as well as computational complexity issues. A tool to design ontology networks allowing modelers to define different relationships, can benefit from the formalization.

\section{Research Methodology}

This work is being carried out following an iterative process. I started with the analysis of case studies to identify ontology relationships. This led to investigate the way other authors addressed this issue, reviewing theoretical foundations about DL and computational complexity when necessary. As a result, a set of relationship definitions is obtained, which is validated in a case study, and from the weakenesses found a new iteration starts, refining the previous definitions.

Regarding the evaluation of the approach, the implementation of an application to design ontology networks is being carried out. It will allow to validate a lot of important aspects: (i) its usability to define different relationships, reaching the adecuate abstraction level (ii) the evaluation of the user satisfaction when the ontology network evolves. Here, it is important to know about the imposed restrictions for ensuring the ontology network consistency: if they help at the moment of introducing changes or they difficult the task in practice.

\section{Results}

I have formalized four ontology relationships, introduced in Section 3. A first formalization and its use to describe a web recommender system was presented in [11. In the following, I present the usesSymbols Of relationship.

First, I define a relationship between two ontologies $O$ and $O^{\prime}$ w.r.t. a query language $\mathcal{Q} \mathcal{L}$ as a set of axioms $A_{r}$, called relationship axioms such that:

$A_{r} \subseteq\left\{\alpha \in \mathcal{Q L} \mid \operatorname{sig}(\alpha) \subseteq \operatorname{sig}(O) \cup \operatorname{sig}\left(O^{\prime}\right) \cup S_{r}\right\}$ where

$S_{r} \subseteq\left\{X \mid X \in N_{C} \cup N_{R} \cup N_{I}\right\}$ is called the relationship signature with:

$N_{C}$ the set of all the concept names, $N_{R}$ the set of all the role names, $N_{I}$ the set of all the individual names

$$
S_{r} \cap \operatorname{sig}\left(O \cup O^{\prime}\right)=\varnothing
$$


uses SymbolsOf $\left(O, O^{\prime}, \mathcal{Q} \mathcal{L}\right)$ is defined by a set of relationship axioms $A_{r}$ such that:

$S_{r} \subseteq\left\{r \mid r \in N_{R}\right\}$ is the relationship signature, $\operatorname{sig}(O) \cap \operatorname{sig}\left(O^{\prime}\right)=\varnothing$

$A_{r} \subseteq\left\{r(i, j) \mid r \in S_{r}, i \in N_{I} \cap \operatorname{sig}(O), j \in N_{I} \cap \operatorname{sig}\left(O^{\prime}\right)\right\} \cup\{A \sqsubseteq C \mid A \in$ $N_{C} \cap \operatorname{sig}(O), C$ is a concept description of one of the forms: $\exists r . B, \forall r . B, \geq n r . B$ with $r \in S_{r}, B \in N_{C} \cap \operatorname{sig}\left(O^{\prime}\right), n$ a natural number $\}, A_{r} \neq \varnothing$

$O \cup A_{r}$ and $O^{\prime}$ are S-inseparable w.r.t. $\mathcal{Q} \mathcal{L}$ for $S=\operatorname{sig}\left(A_{r}\right) \cap \operatorname{sig}\left(O^{\prime}\right)$

$O \cup A_{r}$ and $O$ are $\mathrm{S}$-inseparable w.r.t. $\mathcal{Q} \mathcal{L}$ for $S=\operatorname{sig}(O)$

The two last statements ensure the consistency, preventing contradictory results over the symbols of $O^{\prime}$ being used and over the ontology $O$, extended by the set of axioms $A_{r}$.

These relationships have been adressed by different authors separately, not always related to ontologies, some of them taking care of the logical consistency. This work intend to uniformly address the definition of a set of ontology relationships, enough to describe a real application, ensuring its consistency without neglecting complexity issues. I think this work will contribute to the definition of a methodology to design ontology networks.

\section{References}

1. Allocca, C., D'Aquin, M., Motta, E.: DOOR - Towards a Formalization of Ontology Relations. In: Dietz, J.L.G. (ed.) KEOD, pp. 13-20. INSTICC Press (2009)

2. Cuenca Grau, B., Parsia, B., Sirin, E.: Combining OWL Ontologies Using $\varepsilon-$ Connections. Web Semantics: Science, Services and Agents on the World Wide Web 4, 40-59 (2006)

3. Cuenca Grau, B., Horrocks, I., Kazakov, Y., Sattler, U.: Modular Reuse of Ontologies: Theory and Practice. J. Artif. Intell. Res (JAIR) 31, 273-318 (2008)

4. Konev, B., Lutz, C., Walther, D., Wolter, F.: Formal Properties of Modularisation. In: Stuckenschmidt, H., Parent, C., Spaccapietra, S. (eds.) Modular Ontologies. LNCS, vol. 5445, pp. 25-66. Springer, Heidelberg (2009)

5. Borgida, A., Serafini, L.: Distributed Description Logics: Assimilating Information from Peer Sources. Journal of Data Semantics 1, 153-184 (2003)

6. Giunchiglia, F., Walsh, T.: A Theory of Abstraction. Journal Artificial Intelligence 56, 323-390 (1992)

7. Ehrig, M.: Ontology Alignment. Bridging the Semantic Gap. Springer Science+Business Media, LLC (2007)

8. Suchanek, F.M., Abiteboul, S., Senellart, P.: Ontology Alignment at the Instance and Schema Level. Technical report, Institut National de Recherche en Informatique et en Automatique (2011)

9. Baader, F., Nutt, W.: Basic Description Logics. In: Baader, et al. [12], pp. 47-95

10. Donini, F.M.: Complexity of Reasoning. In: Baader, et al. [12], pp. 101-138

11. Díaz, A., Motz, R., Rohrer, E.: Making Ontology Relationships Explicit in a Ontology Network. In: The V Alberto Mendelzon International Workshop on Foundations of Data Management (May 2011)

12. Baader, F., Calvanese, D., McGuinness, D.L., Nardi, D., Patel-Schneider, P.F. (eds.): The Description Logic Handbook: Theory, Implementation, and Applications. Cambridge University Press (2003) 